\title{
A Few More Heteronyms/Heterophones to Buttress the Argument That Yoruba is a Language Isolate
}

\author{
Mister Seun Ayoade* \\ Independent Researcher, Winner 2004 NIIT Scholarship, Nigeria
}

Submission: May 05, 2019; Published: May 13, 2019

*Corresponding author: Mister Seun Ayoade, Independent Researcher, Winner 2004 NIIT Scholarship, Nigeria

\section{Mini Review}

"The Yoruba language is very musical....to distinguish the tones thus becomes very indispensable" [1]."In Yoruba, vowels are of greater importance than consonants, and tones than vowels; hence the peculiarity of this language, that musical sounds can be employed to convey a correct idea of words in speech" [2]. "The language however abounds in contractions and elisions; a whole syllable may be dropped but the tone remaining. This is the crux of difficulty with foreigners trying to speak the language. There is no word accent in Yoruba, the tone governs everything.... [3]. "No consonants are used to represent a vowel by perverting them from their legitimate consonanted sounds as ' $h$ ', ' $w$ ' and ' $y$ ' are sometimes used in English [4]. "The labors of Mr. Crowther who reduced the language and collected into a vocabulary several thousand words and of Mr Bowen who increased the number to about eight thousand words show most conclusively its fullness and variety which, in the progress of time, must become more and more manifest as continual study and investiga tion develop its resources. It is capable of almost indefinite expansion and is so constructed that a scholar familiar with all its principles could increase the number of words ad infinitum. It is a language of vowels having but very few consonants and is thus rendered more difficult to acquire, especially those whose language is differently constructed...The language is most difficult because of the innumerable contractions made by the natives in rapid speaking. Not infrequently contracted into three and seven into four and five, so puzzling the learner that he considers it next to an impossibility to catch the words with any distinctness. If, on the other hand he tries to speak it he will oftentimes find himself expressing an idea he never thought of by wrong accent.

The language is abundant in proverbs and such expressions as indicate thought" [5]. A few more examples of Yoruba homographs and heterophones/ heteronyms include the following: kindly note that the alphabet ' $d$ ' stands for the sound 'do', ' $r$ ' stands for the sound 're' and ' $m$ ' for 'mi' (Table 1).

Table 1: A few more examples of Yoruba homographs and heterophones/ heteronyms.

\begin{tabular}{|c|c|c|}
\hline Yoruba Word & Pronunciation & Meaning \\
\hline Pade & $\begin{array}{l}\mathrm{Mm} \\
\mathrm{dm}\end{array}$ & $\begin{array}{l}\text { Shut } \\
\text { meet }\end{array}$ \\
\hline Agbo & $\begin{array}{l}\text { Dd } \\
\mathrm{dr}\end{array}$ & $\begin{array}{c}\text { Ram } \\
\text { Herbal brew }\end{array}$ \\
\hline Alo & $\begin{array}{l}\mathrm{Dm} \\
\mathrm{Rr} \\
\mathrm{rd}\end{array}$ & $\begin{array}{c}\text { Riddle } \\
\text { Going } \\
\text { Something grounds }\end{array}$ \\
\hline Apata & $\begin{array}{l}\text { Dmr } \\
\text { Rrr } \\
\text { drd }\end{array}$ & $\begin{array}{c}\text { Rock } \\
\text { Shield } \\
\text { butcher }\end{array}$ \\
\hline koriko & $\begin{array}{l}\mathrm{Rmr} \\
\mathrm{drd}\end{array}$ & $\begin{array}{l}\text { Grass } \\
\text { Wolf }\end{array}$ \\
\hline Won & $\begin{array}{c}M \\
\mathrm{r}\end{array}$ & $\begin{array}{c}\text { Scarce, rare, expensive } \\
\text { Them }\end{array}$ \\
\hline Osun & $\begin{array}{l}\mathrm{Rd} \\
\mathrm{dr}\end{array}$ & $\begin{array}{l}\text { Camwood, sandalwood } \\
\text { A river, A Yoruba Goddess }\end{array}$ \\
\hline Aba & $\begin{array}{l}\mathrm{Rd} \\
\mathrm{Dm} \\
\mathrm{dd}\end{array}$ & $\begin{array}{c}\text { Hamlet } \\
\text { Suggestion/plan } \\
\text { Ladder }\end{array}$ \\
\hline
\end{tabular}


Global Journal of Archaeology \& Anthropology

\begin{tabular}{|c|c|c|}
\hline Ori & $\begin{array}{l}\mathrm{Rm} \\
\mathrm{Dm}\end{array}$ & $\begin{array}{c}\text { Head } \\
\text { Shea butter }\end{array}$ \\
\hline Ore & $\begin{array}{l}\mathrm{Rr} \\
\mathrm{Rm} \\
\mathrm{Dm}\end{array}$ & $\begin{array}{l}\text { Gift } \\
\text { Cane/whip } \\
\text { Friend }\end{array}$ \\
\hline $\mathrm{Sa}$ & $\begin{array}{c}\text { M } \\
\text { R } \\
\text { M (with accent on 'S') }\end{array}$ & $\begin{array}{l}\text { Run } \\
\text { Hang to dry } \\
\text { fade }\end{array}$ \\
\hline $\mathrm{ku}$ & $\begin{array}{c}M \\
d\end{array}$ & $\begin{array}{c}\text { Die } \\
\text { Remain }\end{array}$ \\
\hline Eni & $\begin{array}{l}\mathrm{Rm} \\
\mathrm{Rr}\end{array}$ & $\begin{array}{c}\text { Mat } \\
\text { Person }\end{array}$ \\
\hline Gbe & $\begin{array}{c}\mathrm{M} \\
\mathrm{R} \\
\mathrm{M} \text { (with accent on 'e') }\end{array}$ & $\begin{array}{l}\text { Dig } \\
\text { Carry } \\
\text { Dry }\end{array}$ \\
\hline Egba & $\begin{array}{l}\mathrm{Rr} \\
\mathrm{Dm} \\
\mathrm{Rd}\end{array}$ & $\begin{array}{l}\text { Cane/whip } \\
\text { A Yoruba tribe } \\
\text { Take! Receive! }\end{array}$ \\
\hline kan & $\begin{array}{l}M \\
D \\
M\end{array}$ & $\begin{array}{c}\text { To break } \\
\text { To touch } \\
\text { To become sour or go off taste }\end{array}$ \\
\hline Asa & $\begin{array}{l}\text { Dm } \\
\text { dd }\end{array}$ & $\begin{array}{c}\text { Eagle } \\
\text { Culture/tradition }\end{array}$ \\
\hline Ede & $\begin{array}{l}\mathrm{Rr} \\
\mathrm{Dd}\end{array}$ & $\begin{array}{c}\text { Name of a Yoruba town } \\
\text { Language }\end{array}$ \\
\hline Elede & $\begin{array}{l}\text { Rmd (with accent on all three 'e's } \\
\text { Rmr } \\
\text { Rmd }\end{array}$ & $\begin{array}{l}\text { Pig } \\
\text { Person from town called "Ede" } \\
\text { Speaker of a certain language }\end{array}$ \\
\hline $\mathrm{Ji}$ & $\mathrm{m}$ & $\begin{array}{l}\text { Wake } \\
\text { steal }\end{array}$ \\
\hline eji & $\begin{array}{l}\mathrm{Rd} \\
\mathrm{dm}\end{array}$ & $\begin{array}{l}\text { Second } \\
\text { gap }\end{array}$ \\
\hline Eje & $\begin{array}{l}\mathrm{Rd} \\
\mathrm{mr}\end{array}$ & $\begin{array}{c}\text { Blood } \\
\text { The numeral } 7\end{array}$ \\
\hline Esan & $\begin{array}{l}\mathrm{Dr} \\
\mathrm{md}\end{array}$ & $\begin{array}{c}\text { Reward, comeuppance } \\
\text { The numeral } 9\end{array}$ \\
\hline Ogbon & $\begin{array}{c}\mathrm{Rm} \\
\mathrm{rd}\end{array}$ & $\begin{array}{c}\text { Intelligence } \\
\text { The numeral } 30\end{array}$ \\
\hline Ogun & $\begin{array}{l}\mathrm{Rm} \\
\mathrm{rd}\end{array}$ & $\begin{array}{c}\text { Eighth month of the year according to Yoruba } \\
\text { calendar } \\
\text { A river }\end{array}$ \\
\hline kere & $\begin{array}{c}\mathrm{Mm} \\
\mathrm{rd}\end{array}$ & $\begin{array}{c}\text { Small } \\
\text { Not intelligent }\end{array}$ \\
\hline kokoro & $\begin{array}{l}\text { Mmm } \\
\text { Mmm (with accents on the three 'o's) } \\
\text { ddd }\end{array}$ & $\begin{array}{c}\text { Tubular snack } \\
\text { Key } \\
\text { Insect }\end{array}$ \\
\hline koro & $\begin{array}{l}\text { Rd (accent on both 'o's) } \\
\text { Rd (no accents on 'o's) }\end{array}$ & $\begin{array}{l}\text { Hidden place } \\
\text { Bitter }\end{array}$ \\
\hline Yiyo & $\begin{array}{l}\mathrm{Md} \\
\mathrm{Mr} \\
\mathrm{Mm}\end{array}$ & $\begin{array}{l}\text { Slippery } \\
\text { Removal } \\
\text { Satiety }\end{array}$ \\
\hline Were & $\begin{array}{c}\text { Mm } \\
\text { Rd } \\
\text { Mm (accent on both 'e's) }\end{array}$ & $\begin{array}{c}\text { An genre of vocal and percussive music } \\
\text { Lunatic } \\
\text { Quickly, suddenly }\end{array}$ \\
\hline Oka & $\begin{array}{l}\mathrm{Rd} \\
\mathrm{Rm}\end{array}$ & $\begin{array}{l}\text { Brew, porridge } \\
\text { Python }\end{array}$ \\
\hline
\end{tabular}


Global Journal of Archaeology \& Anthropology

\begin{tabular}{|c|c|c|}
\hline wo & $\begin{array}{c}\mathrm{D} \\
\mathrm{R} \\
\mathrm{M} \\
\mathrm{M} \text { (with accent below 'o') }\end{array}$ & $\begin{array}{c}\text { Watch } \\
\text { Enter } \\
\text { Demolish } \\
\text { Skewed/tilted }\end{array}$ \\
\hline $\mathrm{Fi}$ & $\begin{array}{l}\mathrm{D} \\
\mathrm{m}\end{array}$ & $\begin{array}{l}\text { Tremble/shake } \\
\text { put }\end{array}$ \\
\hline Ero & $\begin{array}{l}\mathrm{Dr} \\
\mathrm{Rd}\end{array}$ & $\begin{array}{c}\text { Engine } \\
\text { Thought/thinking }\end{array}$ \\
\hline Agba & $\begin{array}{l}\mathrm{Rr} \\
\mathrm{Dd} \\
\mathrm{Dm}\end{array}$ & $\begin{array}{l}\text { Rope } \\
\text { Adult/Senior citizen } \\
\text { Canon }\end{array}$ \\
\hline Ayan & $\begin{array}{l}\mathrm{Mr} \\
\mathrm{Rm} \\
\mathrm{dm}\end{array}$ & $\begin{array}{l}\text { Anxiety, nervousness } \\
\text { Cockroach } \\
\text { Hardwood }\end{array}$ \\
\hline Baba & $\begin{array}{l}\mathrm{Dm} \\
\mathrm{Mm} \\
\mathrm{Dd}\end{array}$ & $\begin{array}{l}\text { Father } \\
\text { Filled up } \\
\text { Guinea corn }\end{array}$ \\
\hline eri & $\begin{array}{l}\text { Rd } \\
\text { Rd (with accent on 'e' } \\
\text { rm }\end{array}$ & $\begin{array}{l}\text { Cornchaff } \\
\text { Dirt } \\
\text { head }\end{array}$ \\
\hline Iko & $\begin{array}{l}\mathrm{Rm} \\
\mathrm{Rd} \\
\mathrm{dm}\end{array}$ & $\begin{array}{c}\text { Cough } \\
\text { State messenger } \\
\text { Hook, suspension from hook }\end{array}$ \\
\hline Bata & $\begin{array}{r}\mathrm{Dd} \\
\mathrm{dm}\end{array}$ & $\begin{array}{c}\text { Shoe } \\
\text { Drum/type of dance associated with said drum }\end{array}$ \\
\hline Awo & $\begin{array}{c}\mathrm{Rm} \\
\mathrm{Rd} \\
\mathrm{Rr} \\
\mathrm{Dm} \\
\mathrm{Rd} \text { (accent on 'o') } \\
\end{array}$ & $\begin{array}{c}\text { Guinea fowl } \\
\text { Fishing net } \\
\text { Secret } \\
\text { Crash } \\
\text { Hue/tint/coloration }\end{array}$ \\
\hline darandaran & $\begin{array}{l}\text { Rrrr } \\
\text { mdmd }\end{array}$ & $\begin{array}{l}\text { Shepherd/pastoralism } \\
\text { criminal }\end{array}$ \\
\hline rinrin & $\begin{array}{c}\mathrm{Md} \\
\mathrm{rr}\end{array}$ & $\begin{array}{c}\text { Walking } \\
\text { Saturated/soaking/sodden }\end{array}$ \\
\hline ijo & $\begin{array}{c}\mathrm{Rm} \\
\mathrm{dr}\end{array}$ & $\begin{array}{c}\text { Dancing } \\
\text { Assembly/congregation/church }\end{array}$ \\
\hline ajo & $\begin{array}{l}\mathrm{Rd} \\
\mathrm{dr}\end{array}$ & $\begin{array}{l}\text { Journey } \\
\text { Common purse }\end{array}$ \\
\hline ayo & $\begin{array}{c}\mathrm{Rd} \\
\mathrm{Rd}(\text { ascent on 'o') }\end{array}$ & $\begin{array}{l}\text { Board game } \\
\text { joy }\end{array}$ \\
\hline odo & $\begin{array}{l}\mathrm{Rd} \\
\mathrm{Dd} \\
\mathrm{dr}\end{array}$ & $\begin{array}{c}\text { Stream } \\
\text { Place } \\
\text { The numeral zero }\end{array}$ \\
\hline Riro & $\begin{array}{l}\text { Md } \\
\text { Mr (with accent on 'o' } \\
\text { Mr } \\
\text { Md (with accent on 'o') }\end{array}$ & $\begin{array}{c}\text { Stirred } \\
\text { Metal working } \\
\text { Ache/pain } \\
\text { soft }\end{array}$ \\
\hline eto & $\begin{array}{l}\mathrm{Rd} \\
\mathrm{dm}\end{array}$ & $\begin{array}{c}\text { Plan/schedule/process/procedure } \\
\text { Rights, inheritance }\end{array}$ \\
\hline ito & $\begin{array}{l}\mathrm{Rm} \\
\mathrm{Rd}\end{array}$ & $\begin{array}{l}\text { Saliva/sputum } \\
\text { urine }\end{array}$ \\
\hline $\mathrm{Aje}$ & $\begin{array}{l}\mathrm{Dm} \\
\mathrm{rm}\end{array}$ & $\begin{array}{l}\text { Witch/sorceress } \\
\text { Trade }\end{array}$ \\
\hline ete & $\begin{array}{c}\text { Rd } \\
\text { Rd (with accents on both 'e's }\end{array}$ & $\begin{array}{l}\text { Lips } \\
\text { leprosy }\end{array}$ \\
\hline oju & $\begin{array}{l}\mathrm{Rm} \\
\mathrm{mr}\end{array}$ & $\begin{array}{l}\text { Eye } \\
\text { Greater than }\end{array}$ \\
\hline
\end{tabular}


Global Journal of Archaeology \& Anthropology

\begin{tabular}{|c|c|c|}
\hline idi & $\begin{array}{l}\mathrm{Dm} \\
\mathrm{Rd} \\
\mathrm{Dr}\end{array}$ & $\begin{array}{l}\text { Reason, buttocks } \\
\text { Eagle } \\
\text { Bunch of fruits }\end{array}$ \\
\hline eku & $\begin{array}{l}\mathrm{Rr} \\
\mathrm{rm}\end{array}$ & $\begin{array}{c}\text { Rat } \\
\text { A greeting }\end{array}$ \\
\hline ife & $\begin{array}{c}\mathrm{Mm} \\
\mathrm{dm}\end{array}$ & $\begin{array}{c}\text { Whistle } \\
\text { Love/desire/will }\end{array}$ \\
\hline ebe & $\begin{array}{l}\mathrm{Rd} \\
\mathrm{Dr} \\
\mathrm{dd}\end{array}$ & $\begin{array}{l}\text { Heap } \\
\text { Porridge } \\
\text { pleading }\end{array}$ \\
\hline tete & $\begin{array}{l}\text { Dd } \\
\text { rd }\end{array}$ & $\begin{array}{c}\text { Speedily } \\
\text { Kind of vegetable }\end{array}$ \\
\hline iwo & $\begin{array}{c}\mathrm{Dr} \\
\mathrm{Rd} \\
\mathrm{Dm} \\
\text { Rd (with accent on 'o') }\end{array}$ & $\begin{array}{c}\text { You, yourself } \\
\text { To watch/view } \\
\text { Name of a Yoruba town } \\
\text { To wear clothes/jewelry etc }\end{array}$ \\
\hline sisan & $\begin{array}{l}\mathrm{Md} \\
\mathrm{mr}\end{array}$ & $\begin{array}{c}\text { Watery } \\
\text { payment }\end{array}$ \\
\hline Apa & $\begin{array}{l}\mathrm{Rm} \\
\mathrm{Rd} \\
\mathrm{dm}\end{array}$ & $\begin{array}{c}\text { Arm } \\
\text { Prodigal/miscreant } \\
\text { scar }\end{array}$ \\
\hline lilo & $\begin{array}{l}\mathrm{Mr} \\
\mathrm{md}\end{array}$ & $\begin{array}{l}\text { To go } \\
\text { To use }\end{array}$ \\
\hline otito & $\begin{array}{l}\text { Mrm } \\
\text { dmm }\end{array}$ & $\begin{array}{l}\text { Enough! } \\
\text { truth }\end{array}$ \\
\hline erin & $\begin{array}{c}\mathrm{Dm} \\
\mathrm{rr}\end{array}$ & $\begin{array}{l}\text { Laughter } \\
\text { Elephant }\end{array}$ \\
\hline ola & $\begin{array}{c}\mathrm{Rm} \\
\mathrm{dr}\end{array}$ & $\begin{array}{l}\text { Wealth } \\
\text { Tomorrow, the future }\end{array}$ \\
\hline eti & $\begin{array}{c}\mathrm{Rm} \\
\mathrm{rd}\end{array}$ & $\begin{array}{c}\text { Ear } \\
\text { A day of the week }\end{array}$ \\
\hline titi & $\begin{array}{l}\mathrm{Md} \\
\mathrm{mm}\end{array}$ & $\begin{array}{c}\text { Closed } \\
\text { Evermore/until }\end{array}$ \\
\hline sisi & $\begin{array}{l}\mathrm{Mm} \\
\mathrm{Rm} \\
\mathrm{md}\end{array}$ & $\begin{array}{l}\text { Open } \\
\text { Young lady } \\
\text { Sixpence }\end{array}$ \\
\hline nina & $\begin{array}{l}\mathrm{Mm} \\
\mathrm{md}\end{array}$ & $\begin{array}{l}\text { Spending of money, energy etc } \\
\text { To beat up }\end{array}$ \\
\hline bibi & $\begin{array}{l}\mathrm{Mm} \\
\mathrm{md}\end{array}$ & $\begin{array}{l}\text { To give birth to } \\
\text { To vomit }\end{array}$ \\
\hline eke & $\begin{array}{c}\text { Dm } \\
\text { Dm (no accent on the 'e's) }\end{array}$ & $\begin{array}{l}\text { Cheek } \\
\text { trickery }\end{array}$ \\
\hline aya & $\begin{array}{c}\mathrm{Dd} \\
\mathrm{rr}\end{array}$ & $\begin{array}{c}\text { Chest } \\
\text { Wife/bride }\end{array}$ \\
\hline fo & $\begin{array}{c}\mathrm{D} \\
\mathrm{M} \\
\mathrm{D} \text { (with accent on 'o') }\end{array}$ & $\begin{array}{l}\text { Fly } \\
\text { Break } \\
\text { wash }\end{array}$ \\
\hline ta & $\begin{array}{l}\mathrm{D} \\
\mathrm{r}\end{array}$ & $\begin{array}{c}\text { Sell } \\
\text { peppery }\end{array}$ \\
\hline ki & $\begin{array}{l}\mathrm{R} \\
\mathrm{m}\end{array}$ & $\begin{array}{l}\text { Thick } \\
\text { greet }\end{array}$ \\
\hline esin & $\begin{array}{l}\mathrm{Rr} \\
\mathrm{rd}\end{array}$ & $\begin{array}{l}\text { Horse } \\
\text { religion }\end{array}$ \\
\hline ope & $\begin{array}{l}\mathrm{Dr} \\
\mathrm{rm}\end{array}$ & $\begin{array}{l}\text { Palm oil } \\
\text { praise }\end{array}$ \\
\hline Ale & $\begin{array}{c}\mathrm{Rm} \\
\mathrm{rd}\end{array}$ & $\begin{array}{l}\text { Night } \\
\text { mistress }\end{array}$ \\
\hline
\end{tabular}




\begin{tabular}{|c|c|c|}
\hline oye & $\begin{array}{l}\text { Rd } \\
\text { rm }\end{array}$ & $\begin{array}{c}\text { Chieftaincy } \\
\text { harmattan }\end{array}$ \\
\hline $\mathrm{Na}$ & $\mathrm{M}$ & Spend \\
& $\mathrm{D}$ & Stretch/elongate/tighten \\
\hline
\end{tabular}

Sese dd Fresh, recently, new

Dr get injured/wounded

Lana dd make something possible/create opportunity

$\mathrm{Rm}$ malfunction (especially of equipment/machinery)

Sina md cause a breakthrough

Dm waywardness, wantonness, profligate

\section{References}

3. Ibid Page XXX.

1. Rev O, Vidal E (1852) A Grammar of the Yoruba Language Compiled by Samuel Crowther, together with introductory remarks, by the. London, UK.

2. Reverend Samuel Johnson, George Routelegde (1921) History of The Yorubas from the earliest Times to the beginning of the British protectorate By and sons, London, UK.

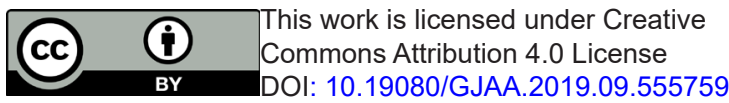

